\title{
THE 30 MICRON EMISSION BAND IN CARBON-RICH PRE-PLANETARY NEBULAE
}

\author{
A. Omont, ${ }^{1}$ S. H. Moseley, ${ }^{2}$ P. Cox ${ }^{3}$ W. Glaccum,${ }^{2,4}$ S. Casey, ${ }^{2}$ T. Forveille, ${ }^{5}$ Kin-Wing Chan, ${ }^{6}$ \\ R. SzCzerba, ${ }^{7}$ R. F. Loewenstein, ${ }^{8}$ P. M. HarVey, ${ }^{9}$ and S. KWOK ${ }^{10}$ \\ Received 1995 January 10; accepted 1995 June 9
}

\begin{abstract}
The 16-48 $\mu \mathrm{m}$ spectra of five carbon-rich post-AGB objects (pre-planetary nebulae, PPNs) known to have an unidentified $21 \mu \mathrm{m}$ emission feature in their IRAS LRS spectra have been obtained using the Kuiper Airborne Observatory. A broad emission band extending from 24 to $\sim 45 \mu \mathrm{m}$ is present in the spectra of these objects. Peaking at $\sim 30 \mu \mathrm{m}$, the intensity of the band is variable from source to source, accounting in one case (IRAS $22272+5435$ ) for $\sim 20 \%$ of its bolometric luminosity. However, its strength is not correlated with that of the $21 \mu \mathrm{m}$ feature. The $30 \mu \mathrm{m}$ band is similar to the feature previously found in other carbon-rich AGB stars and planetary nebulae. It is thus seen in a wide variety of objects, while the $21 \mu \mathrm{m}$ band is seen only in a subset of PPNs. It was suggested for AGB stars that the $30 \mu \mathrm{m}$ band could be carried by solid MgS particles. The observed $30 \mu \mathrm{m}$ emission is modeled combining distributions of carbon grains and MgS grains. The required $\mathrm{MgS}$ abundance with respect to $\mathrm{H}$ nuclei is roughly estimated to be less than $\sim 7 \times 10^{-6}$, i.e., representing about $50 \%$ and $25 \%$ of the total abundances of S and $\mathrm{Mg}$, respectively. Despite the relatively good fit with MgS, the possibility of alternative models especially with carbon-related compounds should still be addressed.
\end{abstract}

Subject headings: circumstellar matter — dust, extinction — infrared: stars - planetary nebulae: general stars: AGB and post-AGB - stars: carbon

\section{INTRODUCTION}

Circumstellar envelopes around evolved stars are by far the most numerous sources detected by $I R A S$ and are the main known sites of dust formation. Among them, those in transition between the asymptotic giant branch (AGB) red giants and planetary nebulae (pre-planetary nebulae, PPNs) are particularly interesting because of their rapid variations in physical conditions. One intriguing class of PPNs ( $21 \mu \mathrm{m}$ PPNs) exhibits a strong unidentified band at $21 \mu \mathrm{m}$ (Kwok, Volk, \& Hrivnak 1989, hereafter KVH). As with other PPNs, their IRAS colors suggest detached envelopes, and they are associated with visible stars of intermediate spectral type (generally G) with supergiant spectral characteristics. Detection of visible $\mathrm{C}_{3}$ bands $(\mathrm{KVH})$ and measurements of large-millimeter $\mathrm{HCN} / \mathrm{CO}$ line intensity ratios (Omont et al. 1993) have shown that they are carbon rich. Most of these properties are similar to those of the archetype PPN, AFGL 2688. However, it is not understood why the IRAS Low Resolution Spectrograph (LRS) spectra of the $21 \mu \mathrm{m}$ PPNs display such strong $21 \mu \mathrm{m}$

\footnotetext{
${ }^{1}$ Institut d'Astrophysique de Paris, C.N.R.S., 98 bis bd. Arago, F-75014 Paris, France.

${ }^{2}$ Laboratory for Astronomy and Solar Physics, Code 685, NASA/Goddard Space Flight Center, Greenbelt, MD 20771.

${ }^{3}$ Observatoire de Marseille, 2 pl. Leverrier, F-13248 Marseille Cedex 04, France. Also Max-Planck-Institut für Radioastronomie, Bonn.

${ }^{4}$ Applied Research Corporation, 8201 Corporate Drive, Landover, MD 20785.

${ }_{5}^{5}$ Observatoire de Grenoble, Université Joseph Fourier and CNRS, B.P. 53X, F-38041 Grenoble Cedex, France.

${ }^{6}$ Astronomy Department, University of Maryland, College Park, MD 20742. Also Laboratory for Astronomy and Solar Physics, NASA/Goddard Space Flight Center.

${ }^{7}$ Department of Physics and Astronomy, The University of Calgary, Alberta T2N 1N4, Canada. Also Institut d'Astrophysique de Paris.

${ }_{8}^{8}$ Yerkes Observatory, Williams Bay, WI 53191.

${ }^{9}$ Astronomy Department, University of Texas, Austin, TX 78712.

${ }^{10}$ Department of Physics and Astronomy, The University of Calgary, Alberta T2N 1N4, Canada.
}

bands as well as equally strong $11-15 \mu \mathrm{m}$ plateaus, while such structures are apparently absent in about half of the known C-rich PPNs or very weak as in the case of AFGL 2688 (Buss et al. 1993). There is also no trace of either feature in C-rich AGB circumstellar envelopes, such as IRC +10216 and AFGL 3068 , or in C-rich planetary nebulae, such as NGC 7027 and IC 418, although the latter sources have an emission band around $11 \mu \mathrm{m}$ attributed to PAHs that could have some relation to the 11-15 $\mu \mathrm{m}$ plateau. The fact that both the $21 \mu \mathrm{m}$ feature and the 11-15 $\mu \mathrm{m}$ plateau are missing in the evolutionary stages preceding and following that of those PPNs is intriguing. This may be related to the transitory state of such objects, which suggests that these features correspond to transient properties of dust in these rapidly varying environments, or a radiative transfer effect.

After the discovery of this class of sources by $\mathrm{KVH}$, a variety of observations in different wavelength ranges, complementary to those of the IRAS LRS, was initiated to improve our understanding of the dust properties in these objects (Buss et al. 1990; Kwok, Hrivnak, \& Geballe 1990; Hrivnak \& Kwok 1991; Omont et al. 1993; Henkel, Cox, \& Omont 1995; Hrivnak 1995).

In this context, we proposed to explore with the Kuiper Airborne Observatory (KAO) the spectral range between 23 and $50 \mu \mathrm{m}$ in order to search for possible additional features that could help to identify the carrier of the $21 \mu \mathrm{m}$ band.

The observations by Buss et al. (1990) have provided confirmation of the unusual properties of the dust in these objects. Their detection of strong emission features in the 6-8 $\mu \mathrm{m}$ wavelength range, together with the weak $3.3 \mu \mathrm{m}$ band detected by Kwok et al. (1990), are reminiscent of polycyclic aromatic hydrocarbon (PAH) emission. However, the ratios between the individual bands are different from those observed in ordinary PAH sources, and there is a wide range in these ratios (Cohen et al. 1986). This fact, together with the need to achieve the excitation with the visible light of these relatively 
cold stars (F or G), lead Buss et al. (1990) to propose that the emitter is rather some hydrogenated solid amorphous carbon (HAC) or cluster of PAHs.

The purpose of this paper is to report and discuss the results of spectral observations in the range $16-48 \mu \mathrm{m}$ on four sources clearly displaying the $21 \mu \mathrm{m}$ feature: IRAS $07134+1005=$ HD $56126=$ SAO 96709 (hereafter IRAS 07134), IRAS $20000+3239$ (hereafter IRAS 20000), IRAS $22272+5435=\mathrm{BD}+54^{\circ} 2787=$ HD $235858=$ SAO 34504 (hereafter IRAS 22272), and IRAS 23304+6147 (hereafter IRAS 23304) and also for AFGL 2688 (the Egg nebula), which shows a weak feature around $21 \mu \mathrm{m}$ (Buss et al. 1993). The main observational result is the presence of a broad emission band around $30 \mu \mathrm{m}$ in all five sources; the strength of this band is variable from source to source and is not correlated with the strength of the $21 \mu \mathrm{m}$ band. Unlike the $21 \mu \mathrm{m}$ emission feature, which has been only detected in PPNs, the $30 \mu \mathrm{m}$ band is also seen in sources both in the AGB and planetary nebula stage (Forrest, Houck, \& McCarthy 1981) which implies that the 30 $\mu \mathrm{m}$ band is not a transient form of dust. The possible identifications for the emitting material of both the 21 and $30 \mu \mathrm{m}$ emission bands will be discussed.

\section{OBSERVATIONS AND RESULTS}

The KAO observations were made during a series of flights in 1989 March and 1990 September with the Goddard Space Flight Center 24 Channel Spectrophotometer (blue Dewar) and in 1992 September with a new version of the instrument, the black Dewar. The observations are summarized in Table 1. For both instruments the beam size is the diameter of a circular aperture in the focal plane, which serves as the entrance slit of the spectrometer. Both instruments have two filter/grating configurations, which run from 16 to $29 \mu \mathrm{m}$ with $0.6 \mu \mathrm{m}$ per channel, and 29 to $48 \mu \mathrm{m}$ with $1.2 \mu \mathrm{m}$ per channel. The measured passband for each channel is nearly square but is strongly affected by interference within the order-sorting filter in the longer wavelength configuration. We measure detector passbands before and after flights. The black Dewar has the additional capability of having its grating angles adjusted during flight.

All observations are calibrated to Mars using Wright's model (1976; Wright \& Odenwald 1983). We correct for atmospheric absorption with FASCOD2 (Clough et al. 1986) and the 1986 HITRAN line compilation (Rothman et al. 1987). A discussion of the data reduction can be found in Glaccum et al. (1987), and a more detailed discussion of the data reduction, systematic errors, and the calibration on Mars can be found in Glaccum (1995). The residual errors from atmosphere correction occurring from 17 to $30 \mu \mathrm{m}$ are $<2 \%$ and $<6 \%$ at longer wavelengths. For most data points the error due to the telluric $\mathrm{H}_{2} \mathrm{O}$ correction is $<1 \%$. At $16 \mu \mathrm{m}$ there is a $10 \%$ residual error from poor correction for telluric $\mathrm{CO}_{2}$ and $\mathrm{N}_{2} \mathrm{O}$ and high sensitivity to pointing errors. The absolute calibration is probably better than $10 \%$ for objects much smaller than the $30^{\prime \prime}$ beam and well centered (Glaccum 1995). The observations of AFGL 2688 were taken at the infrared peak determined by "peaking-up" prior to the measurement. All other objects were measured by centering on the star itself. In all cases, the analysis assumed that both Mars and the target objects are point sources to our beam of $30^{\prime \prime}$.

Given our measured temperatures for these objects, we consider that the assumption of small angular extent is warranted. In the absence of accurate measurements of the far-infrared spatial extent of these objects, there remains an uncertainty in the effect of spatial extent on our inferred source brightness. We believe this effect is likely to be small and that it cannot change the general conclusions of this paper.

Figure 1 displays the $16-48 \mu \mathrm{m}$ spectra obtained with the GFSC 24 Channel Spectrophotometer of the four PPNs clearly showing the $21 \mu \mathrm{m}$ band (Fig. $1 a$ ) and of AFGL 2688 (Fig. 1b). The spectra shown refer to the observing conditions reported in Table 1 . No scaling of the data has been applied. For AFGL 2688, three sets of spectra in the long-wavelength channel are displayed to illustrate the reliability of the present measurements. In the IRAS 07134 spectrum, there are additional errors of less than $5 \%$ point-to-point because of an electronic problem that was not corrected for. The disagreement between the short- and long-wavelength channels in the IRAS 20000 spectrum is the result of a boresight shift that occurred when measuring the short-wavelength channel. The low points at $44.2 \mu \mathrm{m}$ in the spectrum of IRAS 22272 are a poorly corrected $\mathrm{H}_{2} \mathrm{O}$ line, still present despite the fact that great care was given in the $\mathrm{H}_{2} \mathrm{O}$ correction. The feature at $26 \mu \mathrm{m}$ in the IRAS 23304 spectrum is probably not real but due to a notch in the order-sorting filter that caused this channel to be very sensitive to telescope pointing. The poor calibration of the short-wavelength channel in the spectrum of IRAS 23304 is due to a poor boresight that occurred when observing Mars.

TABLE 1

LOG OF OBSERVATIONS

\begin{tabular}{|c|c|c|c|c|c|}
\hline Name & Epoch & Instrument & Grating $(\mu \mathrm{m})$ & Beam Diameter & Position \\
\hline IRAS $07134 \ldots \ldots$ & 1989 Mar 23 & Blue & $\begin{array}{l}16.20-29.58 \\
28.60-48.81\end{array}$ & $\begin{array}{l}30 " \\
30\end{array}$ & $\begin{array}{l}\text { Visible source } \\
\text { Visible source }\end{array}$ \\
\hline IRAS $2000 \ldots \ldots$ & 1992 Sep 10 & Black & $\begin{array}{l}17.42-29.49 \\
16.16-28.06\end{array}$ & $\begin{array}{l}20 \\
20\end{array}$ & $\begin{array}{l}\text { Visible source } \\
\text { Visible source }\end{array}$ \\
\hline & 1992 Sep 14 & Black & $30.78-48.52$ & 20 & Visible source \\
\hline IRAS $22272 \ldots \ldots$ & 1990 Sep 15 & Blue & $\begin{array}{l}16.29-29.15 \\
27.79-48.18\end{array}$ & $\begin{array}{l}30 \\
30\end{array}$ & $\begin{array}{l}\text { Visible source } \\
\text { Visible source }\end{array}$ \\
\hline & 1990 Sep 18 & Blue & $\begin{array}{l}16.29-29.15 \\
27.79-48.18\end{array}$ & $\begin{array}{l}30 \\
30\end{array}$ & $\begin{array}{l}\text { Visible source } \\
\text { Visible source }\end{array}$ \\
\hline IRAS $23304 \ldots \ldots$ & 1990 Sep 15 & Blue & $\begin{array}{l}16.29-29.15 \\
3078-48.52\end{array}$ & 30 & Offset guided $4^{\prime}$ away \\
\hline AFGL $2688 \ldots \ldots$ & 1990 Sep 18 & $\begin{array}{l}\text { Black } \\
\text { Blue }\end{array}$ & $\begin{array}{l}16.29-29.15 \\
27.79-48.18\end{array}$ & $\begin{array}{l}20 \\
30 \\
20\end{array}$ & $\begin{array}{l}\text { VISIDIe source } \\
\text { IR peak } \\
\text { IR peak }\end{array}$ \\
\hline & 1992 Sep 14 & $\begin{array}{l}\text { Black } \\
\text { Black }\end{array}$ & $\begin{array}{l}30.78-48.52 \\
28.69-46.31\end{array}$ & $\begin{array}{l}20 \\
20\end{array}$ & $\begin{array}{l}\text { IR peak } \\
\text { IR peak }\end{array}$ \\
\hline
\end{tabular}



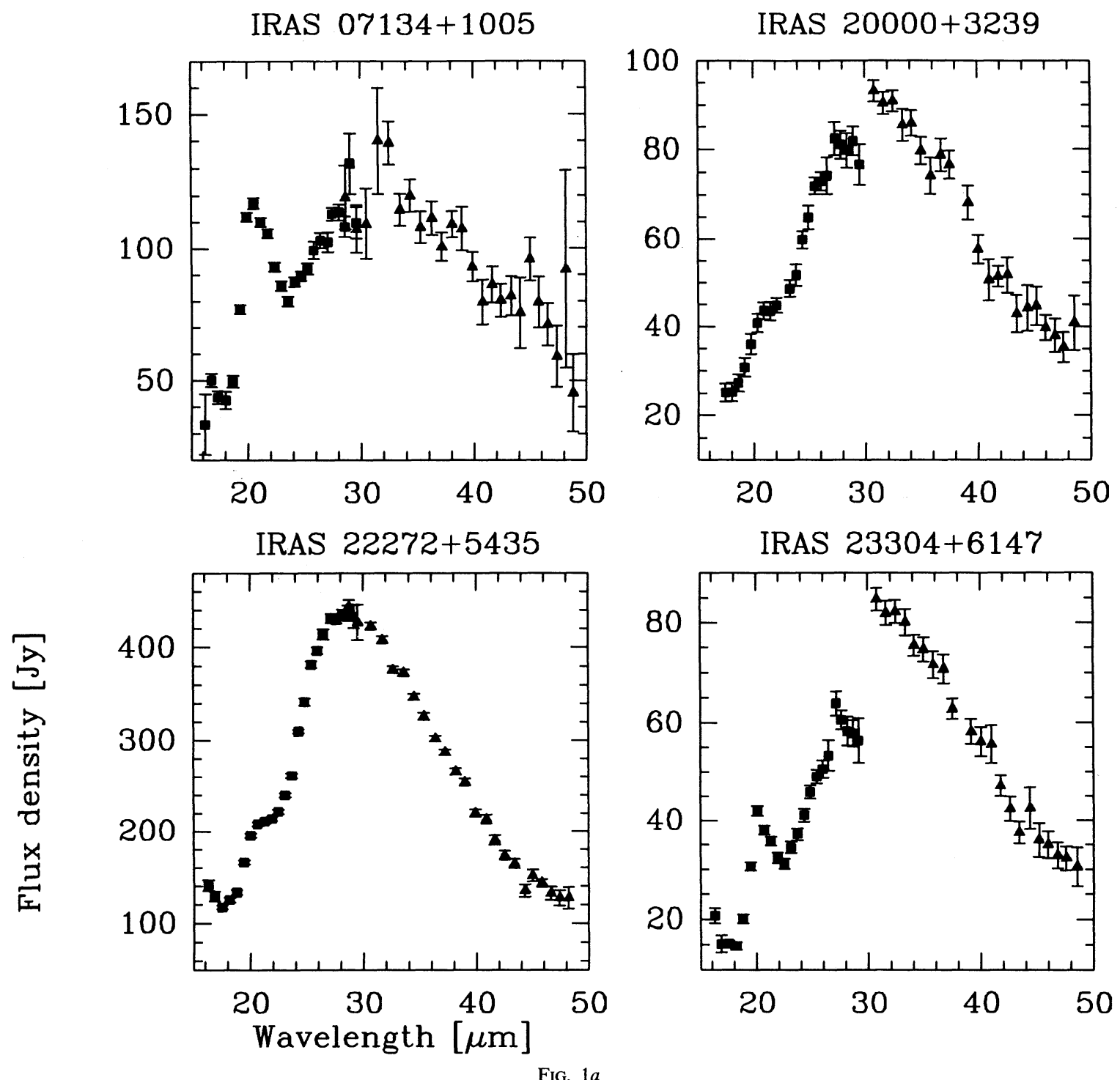

FIG. 1.-(a) The KAO GFSC 24 Channel Spectrophotometer $16-48 \mu \mathrm{m}$ spectra of the four carbon-rich proto-planetary nebulae clearly displaying the $21 \mu \mathrm{m}$ emission feature. The filled squares show the first channel (16-29 $\mu \mathrm{m}, \Delta \lambda=0.6 \mu \mathrm{m})$, and the filled triangles the 30-48 $\mu \mathrm{m}$ channel with $\Delta \lambda=1.2 \mu \mathrm{m}$. No scaling of the data has been applied. The data shown for IRAS 22272 refer to the 1990 September 18 measurements. (b) The 16-48 $\mu$ m spectrum of AFGL 2688 displaying the three sets of spectra obtained in the long-wavelength channel to illustrate the reliability of the present measurements. The 1992 September data have been scaled down, as indicated, for clarity.

Finally, the apparent structure in the $30-48 \mu \mathrm{m}$ spectrum of AFGL 2688 (particularly the apparent 3\% dip at $33 \mu \mathrm{m}$ ) is due to our inability to correct for small pointing errors because of the strong interference fringes in the order-sorting filter.

\section{DISCUSSION}

From the $16-48 \mu \mathrm{m}$ spectra shown in Figures $1 a$ and $1 b$, three facts are clearly observed: (1) the variety of strengths of the $21 \mu \mathrm{m}$ bands, (2) the absence of further narrow features in the $23-48 \mu \mathrm{m}$ range comparable to the $21 \mu \mathrm{m}$ feature, and (3) the rapid rise in emission from $24-25 \mu \mathrm{m}$ to $\sim 30 \mu \mathrm{m}$, which is particularly sharp in the case of IRAS 22272 , with a smoother decline at longer wavelengths. We have checked that the shape of the emission seen from 24 to $\sim 45 \mu \mathrm{m}$ in the four $I R A S$ sources is clearly much narrower than expected from a distribution of dust temperatures, which suggests that a strong emission band is present in this wavelength range. In the case of AFGL 2688, our observations confirm the presence of a very weak emission band at $21 \mu \mathrm{m}$ as suggested by the IRAS LRS data (Buss et al. 1993).

Combining the present data with IRAS LRS spectra and color-corrected IRAS flux densities together with published measurements in the 5-13 $\mu \mathrm{m}$ wavelength range, we show in Figure 2 the composite spectra of the five PPNs from 5 to 48 $\mu \mathrm{m}$. The GSFC 24 Channel Spectrophotometer data of IRAS 07134 have been scaled to the IRAS LRS measurements. In the case of AFGL 2688, we scaled the Buss et al. (1993) and the IRAS LRS data to our 1990 September measurements, which are in good agreement with the 16-24 $\mu \mathrm{m}$ spectrum published by Forrest et al. (1975). The spectra of the five PPNs display a striking series of broad emission bands from 5 to $45 \mu \mathrm{m}$, which are best seen in the spectrum of IRAS 22272. 
AFGL 2688

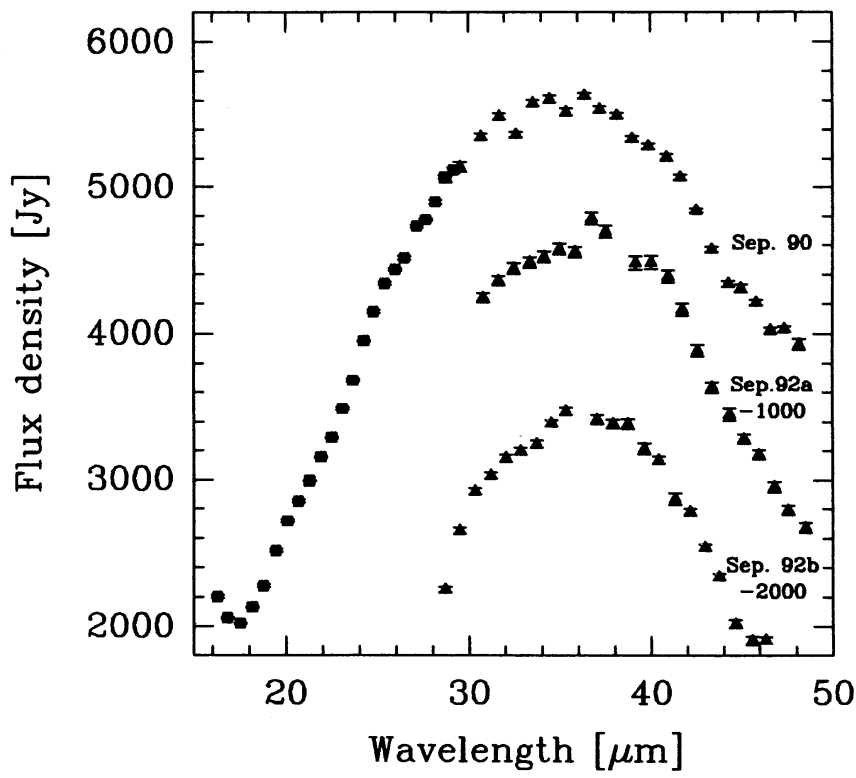

Fig. $1 b$

In order to quantify the characteristics of these broad emission bands, we approximated the continuum emission underlying the data with a sum of two modified blackbodies: $v^{\beta} B_{v}(T)$ at temperatures $T_{1}$ and $T_{2}$ normalized to the data at 10 and 43 $\mu \mathrm{m}$, respectively (Fig. 2). The difficulty is obviously to define the level of this background. We adopted a dust emissivity index of $\beta=1$, appropriate for dust formed in circumstellar envelopes which is thought to be amorphous rather than crystalline (Rouleau \& Martin 1991). In the case of AFGL 2688, Knapp et al. (1994) have shown that the dust emission spectrum is consistent with a $\beta=0.9$ emissivity index from the infrared to the radio spectral range. For all the sources, we achieved reasonable fits to the continuum out to $100 \mu \mathrm{m}$ with temperatures for the dust of 105-120 and 220-280 K (Fig. 2). Shown as dashed lines in Figure 2 is a more quantitative approach to the underlying continuum for the four IRAS sources. A model for AFGL 2688 was not attempted. We used a radiative transfer model based on the code described in Yorke (1980). We assumed the dust to be amorphous carbon (AC) and used the optical constants of the sample AC given in Rouleau \& Martin (1991). A grain size distribution with $n(a) \sim a^{-3.5}$ for $0.005 \leq a \leq 0.25 \mu \mathrm{m}$ was adopted. The circumstellar envelope is represented by a hollow dusty shell expanding at constant velocity with a $r^{-2}$ density law (corresponding to a constant mass-loss rate during the AGB lifetime). A detailed description of the model will appear elsewhere (Szczerba et al. 1995). Both the transfer model and the simplified two-temperature approach provide reasonable approximations for the underlying continuum, but clearly neither of them accounts for the excess emission observed at 30 $\mu \mathrm{m}$ or for the emission bands seen at shorter wavelengths.

Figure 3 presents, for the five PPNs, the infrared excess emission defined as the ratio of the observed spectrum to the underlying continuum (adopting the two-temperature blackbody approximation). A series of four major emission bands in the 5-48 $\mu \mathrm{m}$ wavelength range is seen in Figure 3 in the wavelength ranges $6-9,10-15,19-22$, and $24-45 \mu \mathrm{m}$. The $30 \mu \mathrm{m}$ emission band, extending from 24 to $40 \mu \mathrm{m}$, is the strongest feature, except for IRAS 07134 and IRAS 23304, and accounts, in the case of IRAS 22272 , for $\sim 20 \%$ of its bolometric luminosity. This prominent emission band is detected in all the sources, including AFGL 2688. The relative strengths of the 30 and $21 \mu \mathrm{m}$ emission bands vary strongly from source to source: in the spectra of IRAS 22272 and IRAS 20000 in which the $21 \mu \mathrm{m}$ feature is relatively weak, the $30 \mu \mathrm{m}$ emission band is very strong, and the spectrum of IRAS 07134 , which has a very strong $21 \mu \mathrm{m}$ feature, displays a weak $30 \mu \mathrm{m}$ band. Beside the 21 and $30 \mu \mathrm{m}$ emission bands, there is a broad emission plateau peaking at $11 \mu \mathrm{m}$ and extending out to $15 \mu \mathrm{m}$. This

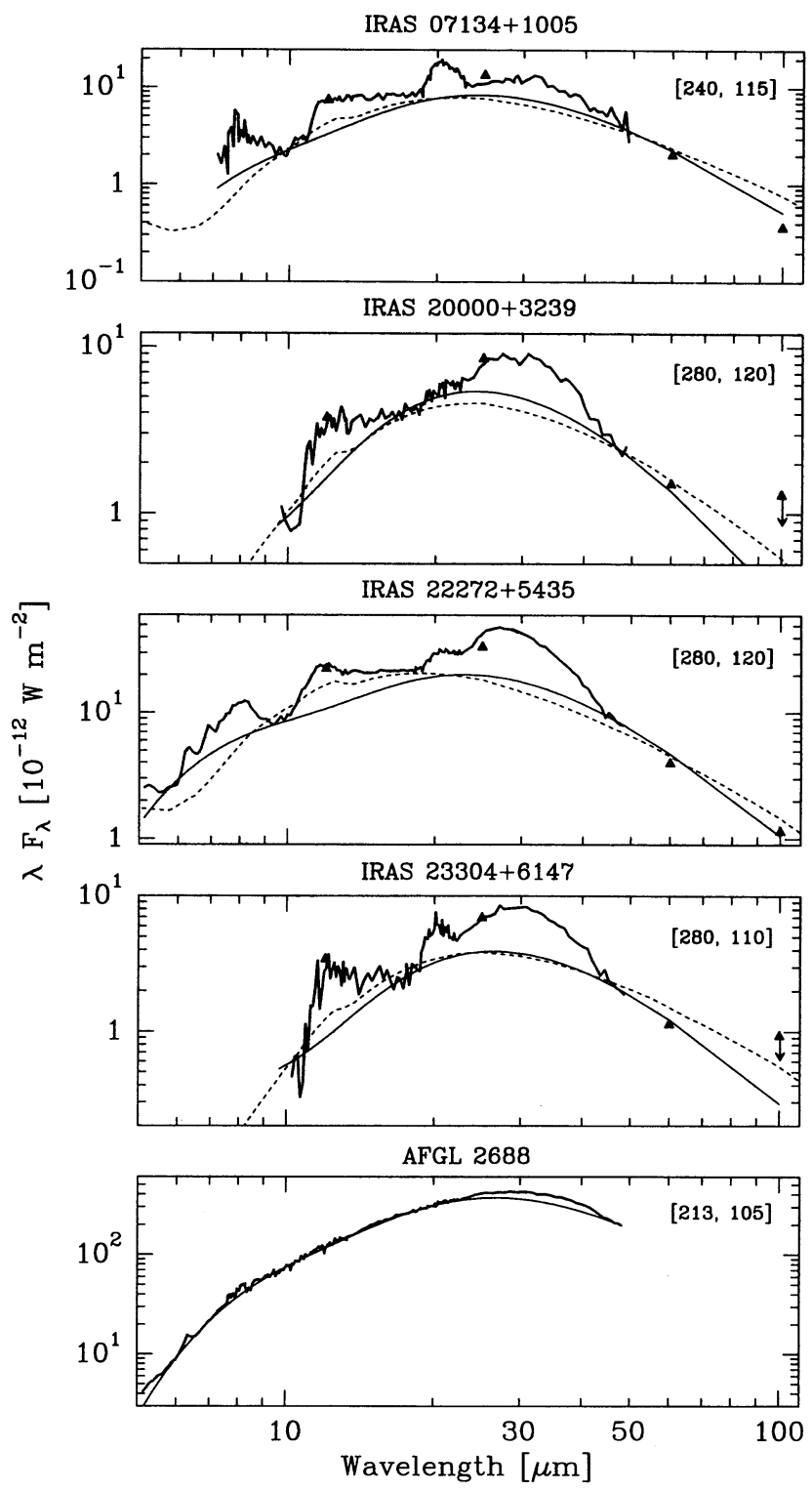

Fig. 2.- Composite infrared spectra of the sources shown in Fig. 1 combining the present KAO GSFC 24 Channel Spectrophotometer data with the IRAS LRS spectra and the IRAS color-corrected photometric data, together with the KAO HIFOGS 5-8 $\mu \mathrm{m}$ spectra of Buss et al. (1990) for IRAS 07134 and IRAS 22272 and the 5-13 $\mu$ m data of Buss et al. (1993) for AFGL 2688. Solid lines represent the emission expected from grains at temperatures $\left[T_{1}, T_{2}\right]$ with emissivities proportional to $\lambda^{-1}$. Dashed lines are the results of a transfer model described in the text. 


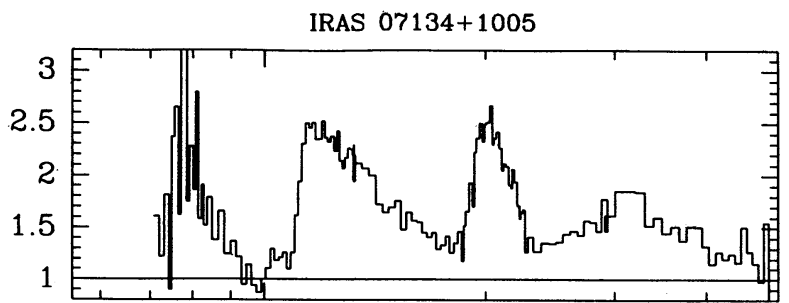

IRAS $20000+3239$
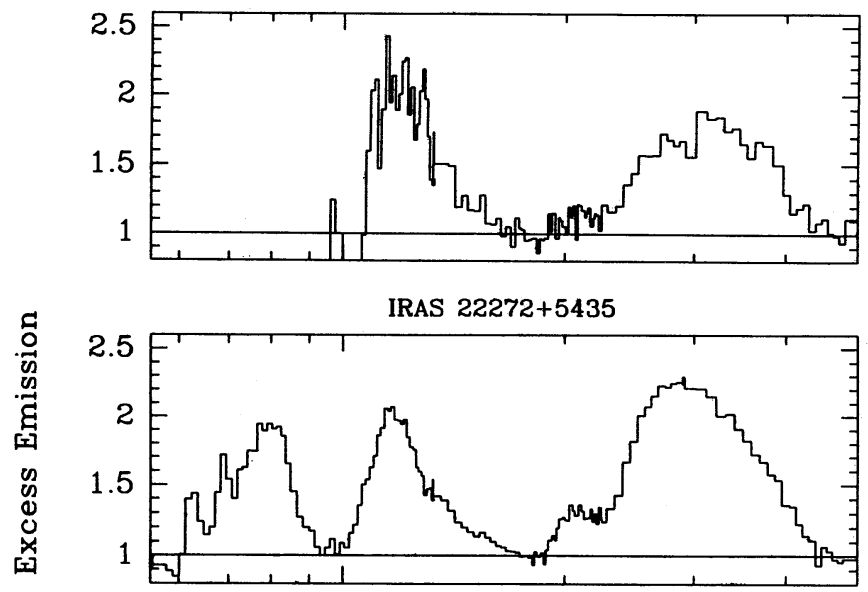

IRAS $23304+6147$
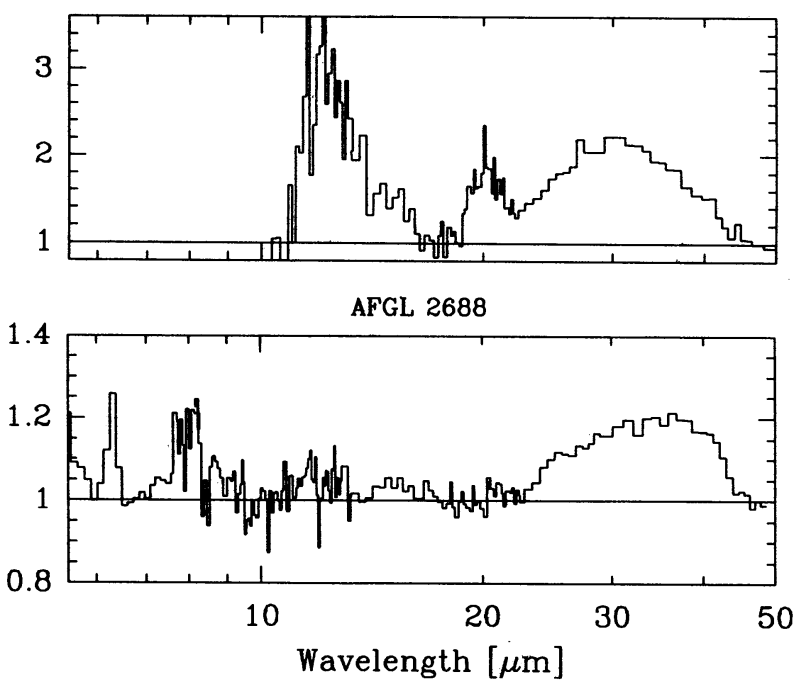

FIG. 3.-Infrared excesses for the five PPNs defined as the ratio of the observed spectrum (shown in Fig. 2) to the two-temperature blackbody approximation of the underlying continuum. Broad emission bands around 7 , 11,21 , and $30 \mu \mathrm{m}$ are clearly seen in the spectra of the PPNs. The relative intensities of these bands change from source to source.

emission is particularly weak in the case of AFGL 2688. For IRAS 22272 and IRAS 07134, the measurements by Buss et al. (1990) show an emission plateau extending from 6 to $9 \mu \mathrm{m}$ and a $6.2 \mu \mathrm{m}$ emission feature attributed to circumstellar PAH molecules. These features are also present in AFGL 2688 together with a newly detected feature at $8.8 \mu \mathrm{m}$ and the weak emission band at $11 \mu \mathrm{m}$ (Buss et al. 1993). In the following, we discuss in turn the possible carriers of the 21 and $30 \mu \mathrm{m}$ emission bands and their relation to the 6-9 $\mu \mathrm{m}$ and 11-15 $\mu \mathrm{m}$ emission plateaus.

\subsection{The 21 Micron Emission Feature}

The present observations have underscored the very broad range of strengths of the $21 \mu \mathrm{m}$ emission feature. This feature is seen only in the short-lived PPN phase and has never been detected in the spectra of carbon-rich AGB stars or planetary nebulae. Its carrier is therefore likely to be a transient species. However, the identification of the emitter remains open. The presence of such a peculiar and transitory emission feature could be related to the extreme conditions to which dust is exposed during the short-lived PPN phase. Several processes at work in PPNs are relevant and should be further explored: high-velocity streaming of grains through the gas, which results in energetic grain-grain collisions (Jura \& Kroto 1990); high-speed stellar winds and molecular flows (Kwok et al. 1990); and rapidly changing radiation fields that depend on the mass-loss rate and the structure of the PPNs.

Various observations, in the visible (Kwok et al. 1990; Hrivnak \& Kwok 1991; Hrivnak 1995), infrared (Buss et al. 1990), and millimeter (Omont et al. 1993; Henkel et al. 1995) spectral regions ranges, have confirmed the carbon richness of the sources exhibiting the $21 \mu \mathrm{m}$ emission feature (which makes oxides improbable as main emitters). The millimeter measurements have shown that the gas composition agrees with a standard C-rich environment, with an $\mathrm{HCN}$ deficiency in IRAS 07134 (Omont et al. 1993; Henkel et al. 1995) definitely ruling out $\mathrm{HNC}$ as the $21 \mu \mathrm{m}$ emitter, as originally proposed in $\mathrm{KVH}$.

Many compounds have vibrational modes around $21 \mu \mathrm{m}$. However, they can be ruled out either because they display other infrared features not present in these sources or because they are unlikely in a carbon-rich environment. The latter argument makes iron oxides as proposed by Cox (1990) improbable. As discussed by Sourisseau, Coddens, \& Papoular (1992), the absence of some characteristic spectral features rules out material such as raw coal or organometallics of the ferrocene class. The possibility of simple amides (also proposed by these authors) appears moderately appealing because of the absence of emission at $3400 \mathrm{~cm}^{-1}$ characteristic of $\mathrm{NH}_{2}$. Goebel (1993) has suggested solid silicon disulfide $\left(\mathrm{SiS}_{2}\right)$ as a possible carrier of the $21 \mu \mathrm{m}$ emission feature based on laboratory measurements by Nuth et al. (1985) which show a broad emission band peaking slightly beyond $22 \mu \mathrm{m}$. Although the fit is not perfect, $\mathrm{SiS}_{2}$ is an interesting candidate (it has no additional infrared bands) and should be given further consideration.

The identification by Buss et al. (1990) of 6-9 $\mu \mathrm{m}$ emission bands in the spectra of the $21 \mu \mathrm{m}$ sources, together with the presence of $11-15 \mu \mathrm{m}$ plateaus, both likely related to HACs or clusters of PAHs, naturally leads us to consider carbonaceous material as a possible carrier of the $21 \mu \mathrm{m}$ emission feature. Although PAHs are known to have vibrational bands in the 15-25 $\mu \mathrm{m}$ range, individual PAHs (such as naphthalene, $\mathrm{C}_{10} \mathrm{H}_{8}$, which has a strong band at $21 \mu \mathrm{m}$ ) are unlikely because the position of their bands depends strongly on the species under consideration. We note that high-resolution spectral measurements of the $21 \mu \mathrm{m}$ feature in IRAS 22272 and IRAS $04296+3429$, which is another PPN showing this feature, do not reveal any further spectral signatures beyond that seen in the low-resolution spectra (Justtanont, Barlow, \& Skinner 1993; Barlow 1993). Moreover, PAHs also display bands that are not observed in the spectra of the $21 \mu \mathrm{m}$ PPNs. The $21 \mu \mathrm{m}$ emission feature could be related to some special formation 
mechanisms of PAHs that lead to noncompact species, which seem to emit preferentially around $21 \mu \mathrm{m}$, instead of compact species which mostly emit at shorter wavelengths $(15-18 \mu \mathrm{m})$. However, it seems difficult to find PAH mixtures not displaying emission bands in the $15-18 \mu \mathrm{m}$ range (L. d'Hendecourt, private communication), which is clearly incompatible with the observations. Further laboratory work is required to see whether the $\mathrm{PAH}$ hypothesis is definitely excluded.

\subsection{The 30 Micron Emission Band}

The main result of our observations is the presence of a 30 $\mu \mathrm{m}$ emission band in all the $21 \mu \mathrm{m}$ PPNs observed so far. The strength of this excess emission is variable from source to source, and it reaches its highest value in the case of IRAS 22272. There is only one other source in which it is as spectacular, the planetary nebula IC 418 (Moseley 1980; Forrest et al. 1981; Moseley \& Silverberg 1986), where the $30 \mu \mathrm{m}$ band represents more than $20 \%$ of the bolometric luminosity. Our observations show that it is a major dust component in many $\mathrm{C}$-rich envelopes. Previous measurements have shown that the $30 \mu \mathrm{m}$ emission band accounts for an important part of the emissivity between 25 and $45 \mu \mathrm{m}$ in prominent cold C-rich envelopes at various stages of evolution including, for instance, the AGB stars IRC + 10216, AFGL 3068 (Forrest et al. 1981; Herter et al. 1982; Goebel \& Moseley 1985), the planetary nebulae IC 418 and NGC 6572 (Forrest et al. 1981), and also NGC 7027 (Moseley et al. 1995). However, we note that none of these sources displays the $21 \mu \mathrm{m}$ band. The absence of the 30 $\mu \mathrm{m}$ emission band in the C-rich sources V Cyg or S Cep (Forrest et al. 1981), which are known to have lower mass-loss rates and hence warm envelopes, could indicate that the carrier of the $30 \mu \mathrm{m}$ band condenses only at low temperature, which implies the need for a large mass-loss rate for its formation.

Measurement over a wide wavelength range is a necessary prerequisite to determine a reliable continuum shape and hence to decide if the $30 \mu \mathrm{m}$ band is present or not. This is particularly true if the band emission is weak or is not clearly distinguished from the continuum emission. This is the case for AFGL 2688 (see above) and for NGC 7027, where the $30 \mu \mathrm{m}$ band was not recognized (McCarthy, Forrest, \& Houck 1978) until sufficient wavelength coverage was obtained (Moseley et al. 1995; see also Cox 1993).

The fact that all the sources in which the $30 \mu \mathrm{m}$ emission band is found are carbon-rich precludes any oxygen-rich species as its carrier. While S-type stars also display a $29 \mu \mathrm{m}$ feature, which could be similar to the feature seen in $\mathrm{C}$-rich sources, most of the O-rich AGB sources that have been observed thus far do not show an excess emission at $30 \mu \mathrm{m}$ (Glaccum 1995). This seems to rule out species such as carbonates, which have a prominent $30 \mu \mathrm{m}$ band (McCarthy et al. 1978) and provide a reasonable fit to the observed $30 \mu \mathrm{m}$ band (L. d'Hendecourt, private communication).

It was first suggested by Goebel \& Moseley (1985; see also Silverberg, Moseley, \& Glaccum 1987), because of the good agreement between the observed spectra of IRC + 10216 and AFGL 3068 and the laboratory measurements by Nuth et al. (1985), that the emitter is solid magnesium sulfide (MgS). However, the strength of the emission in IC 418 and IRAS 22272 creates some abundance constraints, requiring a large fraction of the sulfur and magnesium to be condensed into the grains (see below). The lack of refractive index data for magnesium sulfide prevented any further quantitative modeling of the emission in these sources. Recently, Begemann et al. (1994) (see also Mutschke et al. 1994) tabulated the optical constants of $\mathrm{Mg}-\mathrm{Fe}$ sulfides as derived from laboratory measurements in the wavelength range $10-500 \mu \mathrm{m}$.

As discussed by Begemann et al. (1994), the emissivity of MgS grains depends strongly on the shape and size because of the very large values of the refractive index in the $30 \mu \mathrm{m}$ band. It is relatively easy to find a shape-size distribution of $\mathrm{MgS}$ grains that accurately fits the observed infrared excess in IRC +10216 (see Begemann et al.). Figure 4 presents a model of the emission of IRAS 22272 for a joint distribution of pure MgS and amorphous carbon grains. We assume that both grain populations have the same temperature distribution. The simple case of a continuous mantle of $\mathrm{MgS}$ on spherical grains of amorphous carbon appears to be excluded because it yields two peaks in the emissivity with a minimum at $30 \mu \mathrm{m}$ (Szczerba et al. 1995). Therefore, it seems more appropriate to use a continuous distribution of ellipsoids such as the one labeled $C D E$ in Figure 3 of Mutschke et al. (1994). It is seen that the agreement with the observed $30 \mu \mathrm{m}$ band is reasonable. It could be improved by adjusting the shape-size distribution. The abundance of $\mathrm{MgS}$ (number of MgS molecules with respect to the total number of $\mathrm{H}$ nuclei) giving the best fit in Figure 4 is estimated to be $\sim 7 \times 10^{-6}$. This is not an unlikely value since it represents $\sim 50 \%$ of the available sulfur and $25 \%$ of the magnesium for cosmic abundances. However, we note that the optical constants of MgS have not yet been measured at visible and near-infrared wavelengths. This makes a precise estimate of both the temperature and the mass of $\mathrm{MgS}$ difficult. In particular, the visible and near-infrared absorption could be significantly enhanced, requiring even less MgS to explain the observed $30 \mu \mathrm{m}$ feature.

One should also carefully consider the cases for various carbonaceous material which remain viable alternatives since they are a major component of the dust population, with a variety of possible structures and compositions. In this context, it is interesting to note that graphite has a band around $30 \mu \mathrm{m}$ (see Draine \& Lee 1984) that Baron et al. (1987) suggested is connected with the observed $30 \mu \mathrm{m}$ band. We tested this suggestion by including graphite in the transfer models mentioned above and using the optical constants as tabulated in Draine \& Lee (1984). However, the band due to graphite barely stands out from the underlying continuum and thus cannot account for the observed excess. Chaudret et al. (1991) proposed that organo-metallic particles (Fe stuck to small polycyclic aromatic compounds) could exist in the interstellar medium. It is

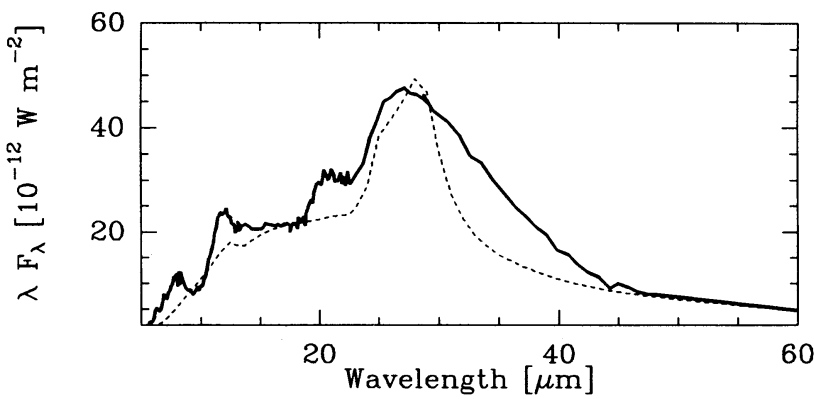

FIG. 4.-The 5-48 $\mu \mathrm{m}$ spectrum of IRAS 22272 compared to the results of a transfer model (dashed line) that includes a joint distribution of $\mathrm{MgS}$ and amorphous carbon grains. Assumptions about the size distribution, shape, and temperature of the $\mathrm{MgS}$ grains are given in the text. 
known from laboratory measurements that metal-carbon stretches are expected to appear near $300-400 \mathrm{~cm}^{-2}$ (McAdams 1967). While the stability of such Fe-PAH complexes may be questionable in the presence of ultraviolet radiation, the case of $\mathrm{Fe}$ stuck to small graphitic or hydrogenated amorphous carbon platelets should be seriously considered. However, Boissel (1994) has recently shown that stable Fe-PAH complexes easily form in an ion trap, so that it is not impossible that similar compounds could transitorily form in the context of grain processing in PPNs.

\section{CONCLUSION}

We present $16-48 \mu \mathrm{m}$ KAO spectra of five carbon-rich PPNs that show in their IRAS LRS spectra the still unidentified $21 \mu \mathrm{m}$ feature. All four sources, together with AFGL 2688, display a broad emission excess extending from 24 to $\gtrsim 40 \mu \mathrm{m}$. The strength of this $30 \mu \mathrm{m}$ band is variable, being in some instances quite weak (IRAS 07134) whereas in other sources (IRAS 22272) it accounts for $\sim 20 \%$ of the bolometric luminosity. However, its strength is not correlated with that of the $21 \mu \mathrm{m}$ feature, which implies different carriers for both features.
Unlike the $21 \mu \mathrm{m}$ emission feature, which has been only detected in PPNs, the $30 \mu \mathrm{m}$ band is also seen in sources both in the AGB and planetary nebula stage, which implies that the $30 \mu \mathrm{m}$ band is not a transient form of dust. Given the strength of the $30 \mu \mathrm{m}$ band, its carrier has to be an abundant dust component in carbon-rich circumstellar shells and possibly a hitherto overlooked, significant component of interstellar dust as well. Solid MgS provides a reasonable fit to the observed band. With an appropriate choice of shape-size distribution of grains, it is possible to explain both the strength and the shape of the $30 \mu \mathrm{m}$ band with, respectively, $\sim 50 \%$ and $\sim 25 \%$ of the available sulfur and magnesium locked in solid MgS. However, various carbonaceous materials remain as viable alternatives since they are the major component of the dust with a variety of possible structures and composition.

We thank the staff of the KAO for their outstanding support of our observations. We are grateful to Professor T. Henning and B. Begemann for providing us with the MgS laboratory measurements prior to publication. We acknowledge the financial support of the CNRS GdRs ISO and PCMGI.

\section{REFERENCES}

Barlow, M. J. 1993, in ASP Conf. Ser. 47, Astronomical Infrared Spectroscopy: Future Observational Directions, ed. S. Kwok (San Francisco: ASP), 97

Baron, Y., de Muizon, M., Papoular, R. \& Pegourie, B. 1987, A\&A, 186, 271

Begemann, B., Dorschner, J., Henning, T., \& Mutschke, H. 1994, ApJ, 423, L71 Boissel, P. 1994, A\&A, 285, L33

Buss, R. H., Jr., Cohen, M., Tielens, A. G. G. M., Werner, M. W., Bregman, J. D., Witteborn, F. C., Rank, D., \& Sandford, S. 1990, ApJ, 365, L23

Buss, R. H., Jr., Tielens, A. G. G. M., Cohen, M., Werner, M. W., Bregman, J. D., \& Witteborn, F. C. 1993, ApJ, 415, 250

Chaudret, B., Le Beuze, A., Rabaa, H., Saillard, J. Y., \& Serra, G. 1991, New. J. Chem., 15, 791

Clough, S. A., Kneizys, F. X., Shettle, E. P., \& Anderson, G. P. 1986, in Sixth Conference on Atmospheric Radiation (Boston: American Meteorological Society), 141

Cohen, M., Allamandola, L. J., Tielens, A. G. G. M., Bregman, J., Simpson,

J. P., Witteborn, F. C., Wooden, D., \& Rank, D. 1986, ApJ, 302, 737

Cox, P. 1990, A\&A, 236, L1

. 1993, in ASP Conf. Ser. 47, Astronomical Infrared Spectroscopy: Future Observational Directions, ed. S. K wok (San Francisco: ASP), 163

Draine, B. T., \& Lee, H. M. 1984, ApJ, 285, 89

Forrest, W. J., Houck, J. R., \& McCarthy, J. F. 1981, ApJ, 248, 195

Forrest, W. J., Merrill, K. M., Russell, R. W., \& Soifer, B. T. 1975, ApJ, 199, L183

Glaccum, W. J. 1995, in preparation

Glaccum, W. J., Moseley, S. H., Campins, H., \& Lowenstein, R. F. 1987, A\&A, 187,635

Goebel, J. H. 1993, A\&A, 278, 226

Goebel, J. H. \& Moseley, S. H. 1985, ApJ, 290, L35

Henkel, C., Cox, P., \& Omont, A. 1995, in preparation

Herter, T., Briotta, D. A., Gull, G. E., \& Houck, J. R. 1982, ApJ, 259, L25

Hrivnak, B. J. 1995, ApJ, 438, 341
Hrivnak, B. J., \& Kwok, S. 1991, ApJ, 368, 564

Jura, M., \& Kroto, H. 1990, ApJ, 351, 222

Justtanont, K., Barlow, M. J., \& Skinner, C. J. 1993, in IAU Symp. 155 , Planetary Nebulae, ed. R. Weinberger \& A. Acker (Dordrecht: Kluwer), 341

Knapp, G. R., Bowers, P. F., Young, K., \& Phillips, T. G. 1994, ApJ, 429, L33

Kwok, S., Hrivnak, B. J., \& Geballe, T. 1990, ApJ, 360, L23

Kwok, S., Volk, K. M., \& Hrivnak, B. J. 1989, ApJ, 345, L51 (KVH)

McAdams, D. M. 1967, Metal-Ligand and Related Vibrations (London: Arnold)

McCarthy, J. F., Forrest, W. J., \& Houck, J. R. 1978, ApJ, 224, 109

Moseley, S. H. 1980, ApJ, 238, 892

Moseley, S. H., \& Silverberg, R. F. 1986, in Interrelations Among Circumstellar, Interstellar, and Interplanetary Dust, ed. J. E. Nuth III \& R. E. Stencel (NASA Conf. Pub. 2403), 233

Moseley, S. H., et al. 1995, in preparation

Mutschke, H., Begemann, B., Dorschner, J., \& Henning, T. 1994, Infrared Phys., 35, 361

Nuth, J. A., Moseley, S. H., Silverberg, R. F., Goebel, J. H., \& Moore, W. J. 1985, ApJ, 290, L45.

Omont, A., Loup, C., Forveille, T., te Lintel Hekkert, P., Habing, H., \& Sivagnanam, P. 1993, A\&A, 267, 515

Rothman, L. S., et al. 1987, Appl. Opt., 26, 4058

Rouleau, F. \& Martin, P. G. 1991, ApJ, 377, 526

Silverberg, R. F., Moseley, S. H., \& Glaccum, W. 1987, in Interstellar Processes: Contributed Papers, ed. D. J. Hollenbach \& H. A. Thronson (NASA TM-88342), 110

Sourisseau, C., Coddens, G., \& Papoular, R. 1992, A\&A, 254, L1

Szczerba, R., et al. 1995 , in preparation

Wright, E. L. 1976, ApJ, 210, 250

Wright, E. L., \& Odenwald, S. F. 1983, BAAS, 12, 456

Yorke, H. 1980, A\&A, 86, 286 